JOURNAL DE PHYSIQUE IV

Colloque C2, supplément au Journal de Physique III, Volume 5, février 1995

\title{
Dynamical Precursors of Martensitic Transitions
}

W. Petry

Physik Department E13, Technische Universität München, D-85748 Garching, Germany

\begin{abstract}
The phonon dispersion of the high temperature bec phase of the group 3 and 4 metals is characterised by a valley of low energy and strongly damped transverse phonons. The large fluctuations in amplitude related to these phonons probe the lattice potential for displacements towards the close packed product phases into which these materials tend to transform. At the same time they stabilize the open bcc structure via their dominating contribution to the lattice entropy. In pure monoatomic metals these precursors are of purely dynamical nature, only the introduction of defects leads to a static condensation of the displacements. Because the trajectory for the displacive transformation and the elementary jump for self diffusion in these metals point in the same direction, the height of the migration barrier and the tendency of the bcc metals to tansform are related to each other. Whereas, for the bcc $\rightarrow$ close packed transition shuffles and homogenous strains involved in the transition could be identified, such an assignment was not possible for the inverse transition, nor for transitions from close packed $\rightarrow$ close packed structures.
\end{abstract}

\section{INTRODUCTION}

Almost half of the elements condense in the open bcc structure. With very few exceptions these bcc phases transform into a close packed structure at lower temperature or under pressure [1]. In all cases the transitions are displacive and of first order. They are mostly reconstructive in the sense that the high temperature/low pressure or parent phase and the product phase are not related by a group - sub group relation. (Rather, the product phase reconstructs a new structure belonging to a different symmetry group.) This reconstruction is achieved by homogenous lattice distortive strains and/or by shuffles. A lattice distortive strain is a homogenous strain that transforms one lattice into another. A shuffle is a coordinated movement of atoms that produces in itself no lattice distortive deformations but alters the symmetry of the crystal. A shuffle deformation can be expressed by a lattice wave modulation of a short wavelength, typically in the order of one to a few nearest neighbour distances. The lattice correspondence observed between parent and product phase defines a structural unit in the parent phase that under the action of homogeneous strain and/or shuffle transforms into a unit of the product phase. Discerning this lattice correspondence does not mean that the actual trajectory for the displacive deformation is known, many ways through real space produce identical lattice correspondences.

Firstly, the question will be adressed whether the actual trajectory for the displacements during the transition can be deduced from the dynamical response of the lattice. With the dynamical response the lattice vibrations or phonons are meant, where for long wavelength the dispersion is given by the elastic constant $\mathrm{c}_{\mathrm{ij}}$ in the corresponding direction. 
It is expected that the transition occurs in such a direction where low energy phonons or particularly low elastic constants indicate a weak repulsive interaction for displacements. Where in reciprocal space shall we find the low energy phonons? At very short momentum transfer $q$ or at large $q$ close to the Brillouin zone boundaries? These will tell us whether long wavelength shears or short wavelength shuffles dominate the transformation. Following this idea we expect dynamical precursors of transitions which are of first order. Eventually these dynamical anomalies become stronger the closer the temperature approaches the transition. It is interesting to speculate whether these large amplitude phonons freeze to static displacements and thereby cause elastic precursors. Discussion of this question deeply involves the role of defects and nucleation for the transition. Finally we show how the tendency of these elements to transform into close packed structures dominates other physical properties: anomalies in the transport mechanism, stabilisation of the high temperature bcc structure, strongly anharmonic lattice potentials etc.

Metallurgists and physicists do not agree on the unique meaning of what is meant by a martensitic phase transition. For a recent review of some of the principles we refer to [2]. Throughout the paper martensitic transformations are discussed in the more general sense of displacive, diffusionless and first order transitions, irrespective whether shuffles alone or homogeneous lattice distortive strains are used.

Martensitic transformations are mainly studied in binary or tertiary alloys, particularly if the shape memory effect is of primary interest. However, if the basic physical properties are addressed, it is extremely helpful to return to elementary systems. In general the structural properties of monoatomic solids are better defined. The influence of all kinds of defects can be thoroughly controlled and phenomena induced by host-defect interactions are expected to be of minor importance. Further, quite a number of martensitic transitions in the elementary systems occur at high temperatures, i.e. thermal equilibrium during the transition is easily achieved. The inherent drawback is the difficult experimental access to single crystals in the high temperature phase - this is also the reason why experimental information on high temperature martensitic transitions is scarcely available. Indeed recent progress in studying high temperature martensitic transitions is due to the development of reliable in-situ growth methods of parent phase single crystals during the experiment [3].

Being interested in dynamical properties of the transition, the choice of experimental methods is almost limited to inelastic neutron scattering. Whereas elastic constants are conventionally measured by ultrasonic methods, this is no longer feasable for the high temperature phases. Further, elastic constants contain only direct information about long wavelength shears. Dynamical instabilities towards opposite shuffles of neighbouring planes have to be explored in full reciprocal space which is only accessible through neutron scattering.

\section{THE BCC $\rightarrow$ CLOSE PACKED STRUCTURE TRANSITIONS}

Promiment examples for transitions from the open bcc structure to closest packed structures are the alkali, earth alkali and the group 3 and 4 transition elements. Transitions in $\mathrm{Li}$ or $\mathrm{Na}$ to $9 \mathrm{R}+\mathrm{fcc}$ and $9 \mathrm{R}+\mathrm{hcp}$ occur at $75 \mathrm{~K}$ and $35 \mathrm{~K}$, respectively. These were amoung the earliest elementary systems in which one was looking for dynamical precursors of the martensitic phase transition. Despite a considerable effort by neutron scattering experiments [4,5] the outcome was to a certain extent disappointing: Dynamical precursors close to the transition temperature are virtually absent. Elastic precursors have been observed but their strength and location in q-space often depend on the thermal history of the crystal. Recent ab initio [6] and thermodynamic [7] calculations describe some of the reasons. Energetically the different closest packed structures are hardly separable and the actual product phase is strongly influenced by the initial crystal quality and thermal history.

The situation is very different in the early transition metals. As indicated in Fig. 1, Sc, Y, La, Ti, Zr and Hf all transform into the hep structure. For the group 4 elements an additional transition to the $\omega$ phase under moderate pressure is known. As an exception, La first transforms to fcc and then to double-hcp. Stassis and co-workers [8] were the first to measure the phonon dispersion of $\beta$ - $\mathrm{Zr}$ by cycling a large $\alpha$-single grain through the $\alpha-\beta$ transition. More detailed measurements could be made by the in-situ growth 
technique developed more recently [9]. Fig. 2 gives a synopsis of the measured phonon dispersions in the parent bcc phase of these elements [10-14].

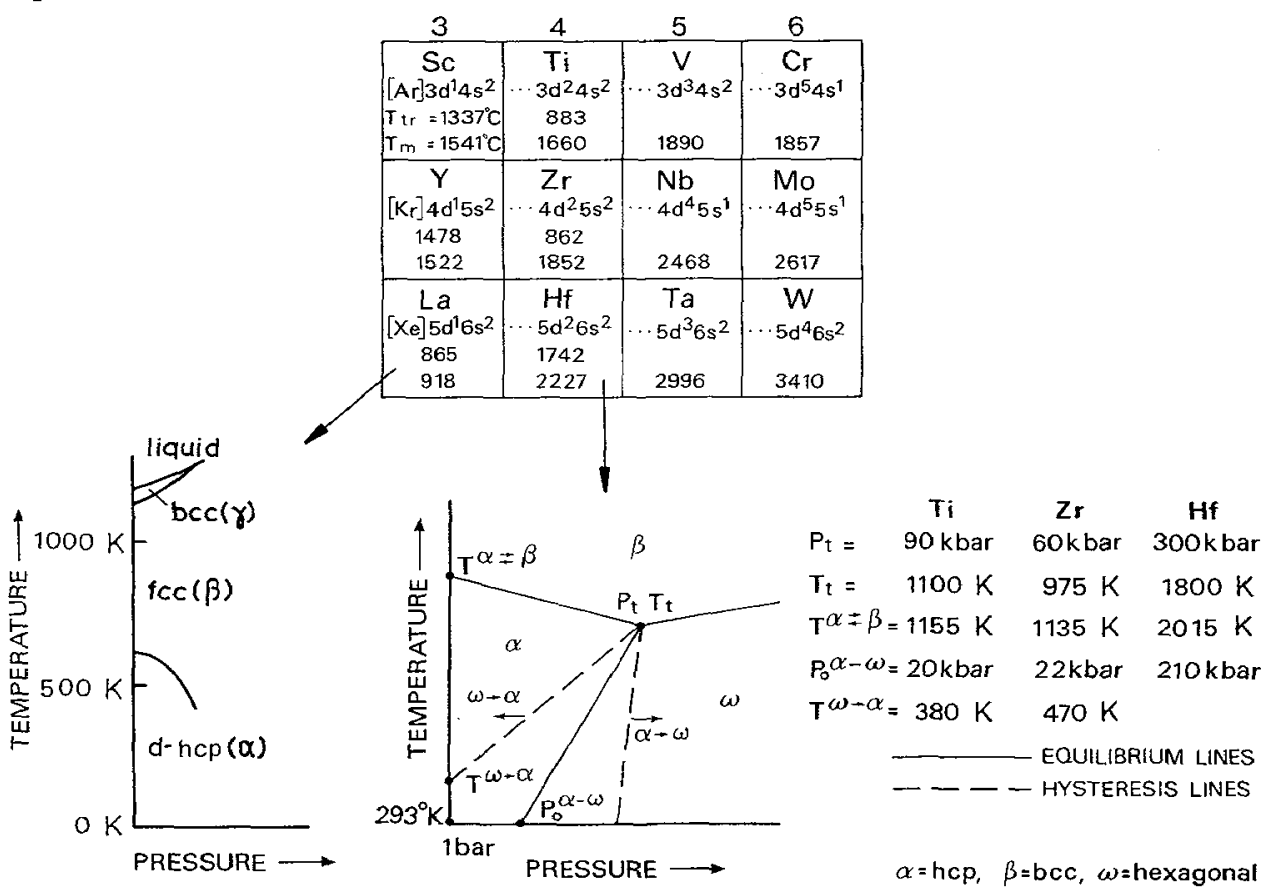

Fig. 1 Transition temperatures $\mathrm{T}_{0}$ and structures for the early transition elements.

The dispersion of the five measured bcc phases greatly resemble one another, i.e. the phonons at higher energy scale roughly with the square root of the mass and the lattice constant, thereby following the homology rule. Most evidently all dispersions are dominated by a few unusual properties: i) At $\xi=2 / 3$ the longitudinal $\mathcal{L}[\xi \xi \xi]$ phonon branch shows a pronounced dip. ii) The whole transverse $T_{1}[\xi \xi 0]$ phonon branch with [1 $\overline{1} 0]$ polarisation is of low frequency when compared to other transverse phonons. iii) The same holds true for the off symmetry $\mathrm{T}_{1}[\xi \xi 2 \xi]$ phonon branch. iv) The low energy phonons are strongly damped. As indicated by the shaded area the line shape of these damped phonons reaches down to zero energy transfer.

\subsection{The bce $\rightarrow \omega$ transition}

The atomic displacements achieved by a longitudinal phonon in $[\xi \xi \xi]$ direction with $\xi=2 / 3$ have a particular crystallographic meaning for the bcc structure. As shown in Fig. 3, for a stationary wave at $\xi=$ $2 / 3$ two of three neighbouring (111) planes move towards each other, whereas every third plane stays at rest. When the two moving planes collapse the perfect $\omega$ structure is achieved. During the second half of the wave, the planes move in the opposite direction and approach the plane at rest. Distortions in these directions are called anti- $\omega$ distortions. From symmetry, it is evident that the restoring forces involved for distortions into the $\omega$ or anti- $\omega$ structure are different.

The $\omega$ lattice can be described as an hexagonal cell, with the $\mathrm{C}$ axis along the $\langle 111\rangle_{\mathrm{bcc}}$ direction and the $\mathrm{A}$ axis along the $\langle 110\rangle_{\text {bec }}$ direction. Thus the crystallographic relation between $\omega$ and $\beta$ reads

$$
(111)_{\mathrm{bcc}} \|(00.1)_{\omega} \text { and }[\overline{1} 01]_{\mathrm{bcc}} \|[01.0]_{\omega}
$$



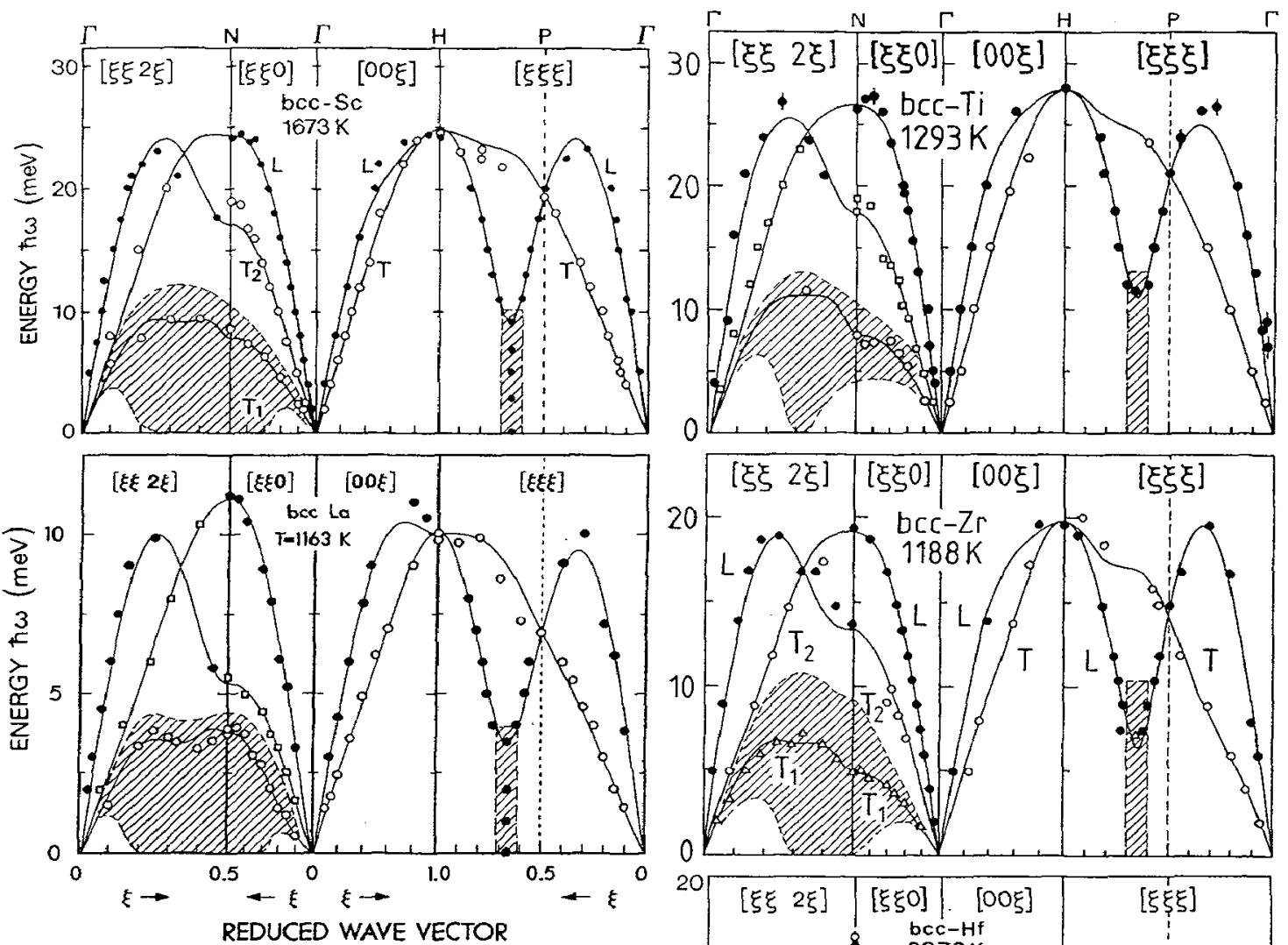

Fig. 2 Measured phonon dispersion in the bcc phase of group 3 and 4 metals. Shaded areas indicate regions of inelastic diffuse scattering or strongly damped phonons. [10-14]

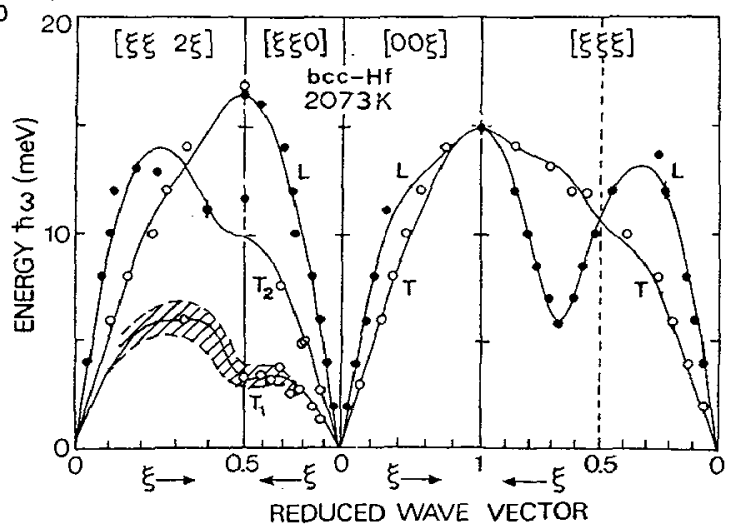

The top spectrum in Fig 4 illustrates what is meant by the shaded area in Fig 2 at L2/3(111). Coherent intensity is measured down to zero (!) energy transfer and even has its maximum at zero energy. Further, the small elastic peak on top of the broad inelastic scattering distribution is of purely incoherent origin and therefore does not represent any coherent elastic scattering. The latter is of particular importance because any static embryo of the $\omega$ phase causes elastic diffuse scattering at $Q=4 / 3(111)$, i.e. at the position where the upper spectrum in Fig. 4 has been measured. Therefore the absence of this "truly" elastic scattering means that within the time window of the method $\left(\leq 10^{-10} \mathrm{~s}\right)$ no stable $\omega$ embryo exists.

Taking resolution effects into account, the broad inelastic distribution can be reproduced by a damped oscillator, the scattering law of which reads

$$
S(Q, \omega)=f(Q) \frac{1}{1-e^{-\hbar \omega / k_{B} T}} \cdot \frac{\Gamma \hbar \omega}{\left(\hbar^{2}\left(\omega^{2}-\omega_{0}^{2}\right)\right)^{2}+(\Gamma \hbar \omega)^{2}}
$$




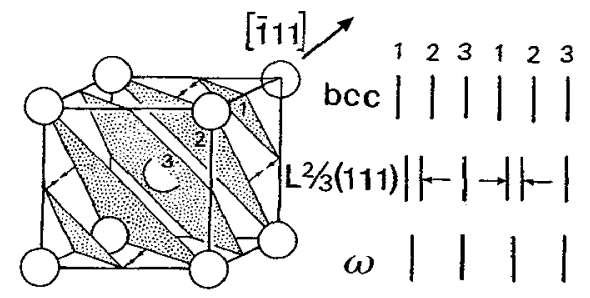

Fig. 3 Scheme of the $b c c \rightarrow \omega$ transition.

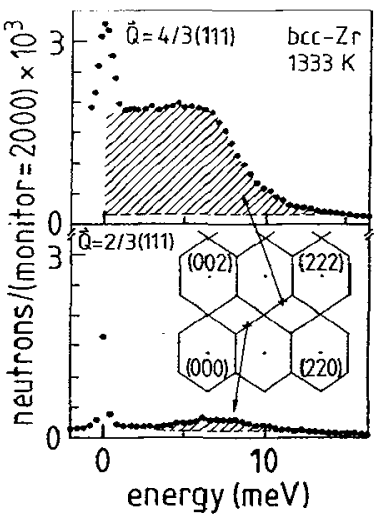

Fig. 4 L2/3(111) phonon measured at two different but equivalent points in reciprocal space. The structure factor $f(Q)$ of the one-phonon scattering law differs by a factor of 1.8 for the two positions [15].

With the exception of $\beta$-Hf, the actual damping term $\Gamma$ observed for the different elements in Fig. 2 is larger than the resonance frequency $\omega_{0}$ itself. This means that the L2/3(111) phonon has an extremely short live time, the amplitude of the corresponding displacement is over-damped after one period of the vibration. This short live time of the L2/3(111) exitation has to be well separated from the question of how this phonon propagates in real space. Along $\mathrm{L}[\xi \xi \xi]$ direction a strong dispersion is observed whereas in the vicinity of $Q=4 / 3(111)$ and in directions perpendicular to $[\xi \xi \xi]$, i.e. in $T[\xi \xi \overline{2 \xi}]$ and $T[\xi \bar{\xi} 0]$ only a very weak dispersion is observed. Translated to real space this means that the displacements corresponding to the L2/3(111) phonon propagate along the [111] chains but neighbouring [111] chains mostly do not follow this displacement. Considering both, the short live time and the localized character of this phonon, propagation perpendicular to [111] is liquid-like.

In the single-oscillator approximation, the amplitude $\mathrm{u}_{0}$ of a vibration is given by

$$
u_{0}=\left(\frac{2 k_{B} T}{m}\right)^{1 / 2} \cdot \frac{1}{\omega_{0}}
$$

The phonon energies at $\mathrm{L} 2 / 3(111)$ are in the order of 3-10 meV corresponding to displacements from 0.4 $0.75 \AA$ at the transition temperature $\mathrm{T}_{0}$. These values are comparable to the displacemenets needed for the $\mathrm{bcc} \rightarrow \omega$ transition. Whereas these large displacements are certainly indicative for the weakness of the bcc lattice towards the $\omega$ transformation their absolute values have to be taken with caution because equ (2) is only a crude approximation.

This unusual behaviour of a monoatomic lattice with a high symmetry structure becomes even more evident when measuring the spectral shape of the $L 2 / 3(111)$ phonon in different Brillouin zones. As shown by the bottom spectrum of Fig. 4 almost no phonon response is measured at $Q=2 / 3(111)$. By no means can this be explained by the $Q$-dependent structure factor $f(Q)$ of the one-phonon scattering law of equ (2), which varies for the actual case of Fig. 4 by a factor of 1.8. An exact explanantion has to take into account interference effects due to phonon-phonon interaction which in the case of over damped phonons strongly alters the one-phonon scattering law [16]. We will not go into detail concerning a quantitative analysis rather present the physical picture which emerges. Because L2/3(111) phonon intensities and line shapes are so different at equivalent points in reciprocal space, we have to conclude that the displacements due to the L2/3(111) phonon locally break the bcc symmetry. (111) Planes fluctuate towards the $\omega$ structure, however during the second half of the wave period amplitudes are so damped that displacements towards the anti- $\omega$ structure are strongly suppressed $[14,15]$. 
Returning to our initial interest that of the bcc $\rightarrow \omega$ transition, we summarise the experimental observations: i) The high temperature bcc phases show large amplitude fluctuations towards the $\omega$ structure. These excitations do not alter with temperature and are also observed in bcc metals of group 3 which do not transfer to the $\omega$ phase. The low restoring force for the shear motion of the [111] chains towards each other is therefore a property inherent to the open bcc structure. For a more fundamental abinitio explanantion on the basis of the electronic configuration, we refer to literature [17]. ii) The L2/3(111) shuffle alone transforms the bcc lattice to a $\omega$ lattice. There is no need for any long wavelength shear. iii) In pure metals no static precursors of the parent phase are observed. As only dynamical precursors exist, the description of a homogenous transition is more appropriate.

\subsection{The bcc $\rightarrow$ hcp transition}

The crystallographic relation for the bcc $\rightarrow$ hcp transition has been established by Burgers [18].

$$
(110)_{\mathrm{bcc}}||(00.1)_{\mathrm{hcp}} \text { and }[\overline{1} 11]_{\mathrm{bcc}} \|[\overline{2} 1.0]_{\mathrm{hcp}}
$$

The transformation can be achieved by the combined displacements of two phonons. The transverse zone boundary phonon $T_{1} 1 / 2(110)$ at the $N$-point with a displacement of neighbouring (110) planes in opposite [1T0] directions by $\delta=a \cdot \sqrt{2} / 12$ achieves the hcp stacking sequence. Two equivalent long wavelength shears - for instance $(1 \overline{1} 2)[\overline{1} 11]$ and $(\overline{1} 12)[1 \overline{1} 1]$ - squeeze the bcc octahedron to a regular hcp one, thereby changing the angle from $109.5^{\circ}$ to $120^{\circ}$. This Burgers mechanism is illustrated in Fig. 5 .

The $T_{1} 1 / 2(110)$ phonons in Fig. 2 are of even lower energies than the so-called $\omega$ phonons, i.e. the bcc lattice exhibits very low restoring forces for shuffling neighbouring (110) planes into opposite [1 $\overline{1} 0$ ] directions. As for the $\omega$ point these phonons are strongly damped. Contrary to what has been observed at the $\omega$ point, the $T_{1} 1 / 2(110)$ phonon energy considerably decreases on approaching the martensitic transition temperature, see Fig. 6. Because the bcc $\rightarrow \omega$ transition is driven by an increase in the pressure, the temperature variation does not alter the related $L 2 / 3(111)$ phonon. The bcc $\rightarrow$ hcp transition however, is driven by temperature and therefore the related $T_{1} 1 / 2(110)$ phonon decreases with temperature as well. But the martensitic transition clearly occurs at finite energy.

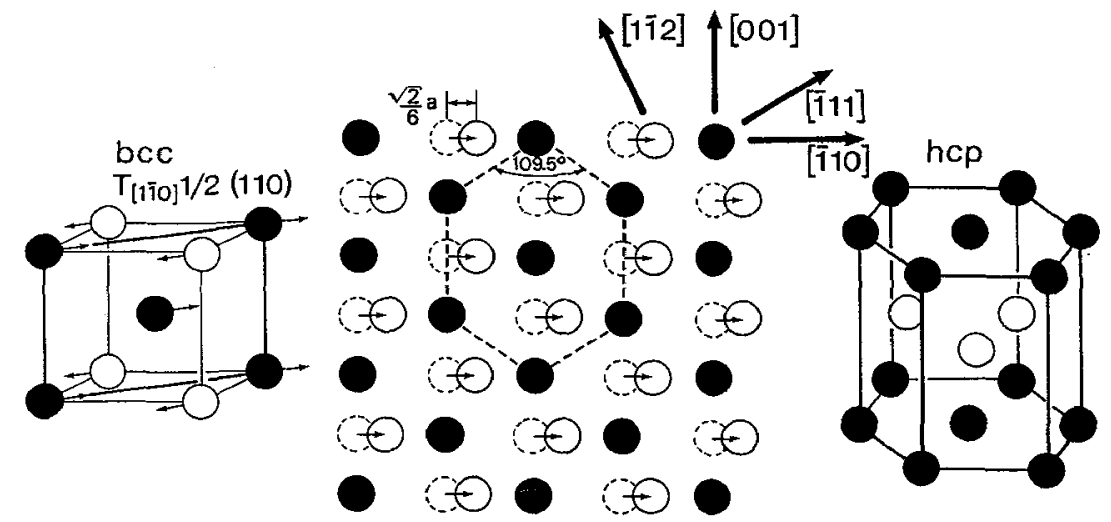

Fig. 5 Scheme of the $b c c \rightarrow$ fcc transition.

The shears needed to complete the transition are those given by the initial slope of the $T_{1}[\xi \xi 2 \xi]$ phonon branch. The elastic constant along this direction is rather low, i.e. one finds low restoring forces for this motion. Recent thermodynamic approaches $[19,20]$, which use an expansion of the free energy in terms of dynamical displacements have suggested that a small softening of the relavant low-energy phonon is sufficient to produce a lower minimum of the free energy required to obtain the product phase. The order parameter in the expansion of free energy is related to the average atomic displacements of the phonons which are associated with the transition. 
Fig. 6 Temperature dependence of the $T_{1} 1 / 2(110)$ phonon in $\beta-\mathrm{Ti}, \beta-\mathrm{Zr}$ and $\beta-\mathrm{Hf}[29]$.

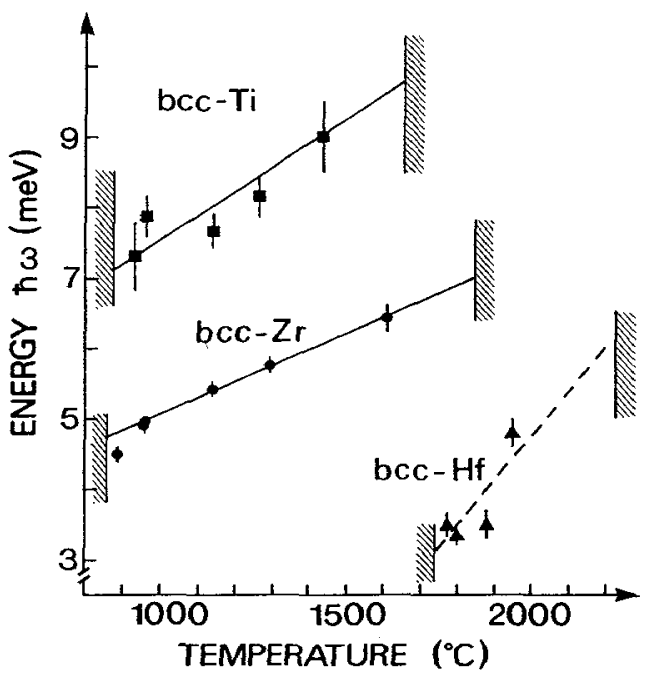

The fact that the $T_{1} 1 / 2(110)$ phonon and the initial slope of the $T_{1}[\xi \xi 2 \xi]$ branch are of little value, do not unequivocally prove that the actual trajectory of the atoms during the transition follow the Burgers mechanism. Computations of energy landscapes may be helpful in filling this argumentive gap. For the pressure induced bcc $\rightarrow$ hcp transition in Ba Chen et al [21] calculated the valley of lowest internal energy as a function of the Burgers shuffle and the homogeneous strain needed to squeeze the octahedron. It turns our that this valley of lowest energy during the combined deformation follows exactly the trajectory assumed in the Burgers mechanism.

We conclude that low frequencies along the $\mathrm{T}_{1}\left[\xi \xi_{0}\right]$ phonon branch which further decrease upon approaching $\mathrm{T}_{0}$ are indicative for the Burgers mechanism. Different to the $\omega$ transition, the bcc $\rightarrow$ hcp transformation needs a combination of a short wavelength shuffle and a homogenous lattice strain.

\subsection{The bcc $\rightarrow$ fcc transition}

Before discussing the $\gamma \rightarrow \beta$ transition in La as an example for the bec $\rightarrow$ fcc transformation, we have to recover some arguments concerning the relation of the phonons involved in the $\omega$ and hcp transition. The longitudinal displacements of the (111) planes for the $\mathrm{L} 2 / 3(111)$ phonon can also be viewed as a shearing of neighbouring [111] rows in opposite directions [22], i.e. $L 2 / 3(111) \equiv \mathrm{T}_{[11 \overline{1}]} 1 / 3(112)$. Noting also the identity $T_{[01 \overline{1}]} 1 / 2(112) \equiv T_{[1 \overline{1} 0]} 1 / 2(110)$ the phonons related to the two above mentioned transitions are no longer isolated points in reciprocal space but are part of the valley of the transverse low energy phonons along $[\xi \xi 2 \xi]$ propagation.

Now, the dispersion of $\gamma$-La shows the same anomalies as the other high temperature bcc phases but instead of transforming to $\omega$ or hcp, it first transforms into fcc. Both fcc and hcp are closest packed structures and differ only by their stacking sequence of the basal plane. As for the bcc $\rightarrow$ hcp transition a short wavelength shuffle shifts the $\mathrm{ABAB}$... sequence of (110) bce planes to an $\mathrm{ABAB}$... sequence of (00.1) hcp planes. But a further $(110)[1 \overline{1} 0]_{b c c}$ long wavelength shear is needed to change to the $A B C A B C .$. sequence of $(111)_{\mathrm{fcc}}$ planes, see Fig. 7. Hence this mechanism is analogous to the Burgers mechanism, as both transitions preserve the interlayer distance of the original (110)bce plane and thus the replusive effect of one layer with respect to the other. The essential difference is that for the bcc $\rightarrow \mathrm{fcc}$ transition a further long wavelength strain of $(110)_{b c c}$ planes into $[1 \overline{1} 0]$ direction is neeeded. The corresponding elastic constant $C^{\prime}=1 / 2\left(C_{11}-C_{12}\right)$ is given by the initial shape of the $T_{1}[110]$ phonon branch. Indeed the Born van Karman analysis of the $\gamma$-La dispersion yields a very low $C^{\prime}$, which is best expressed by the unusual high assymetry parameter for the two shear constants $A=C_{44} / C^{\prime} \cong 10[14]$. 


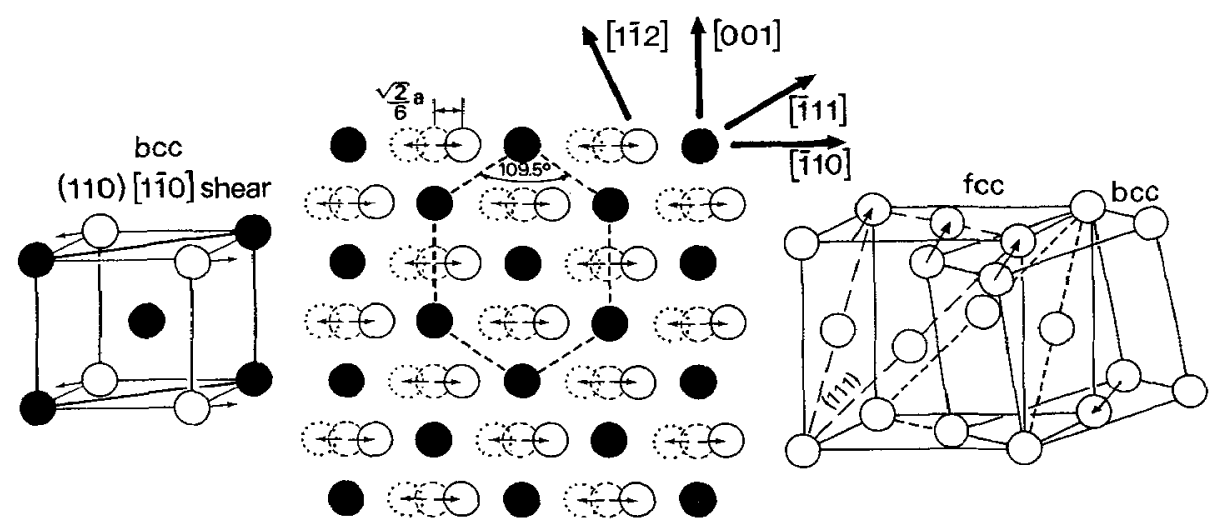

Fig. 7 Scheme of the bcc $\rightarrow$ fcc transition.

The dominating role of $\mathrm{C}^{\prime}$ is confirmed by thermodynamic considerations. Assuming no volume change through the $\gamma \rightarrow \beta$ transition, it can be shown that the local restoring forces tending to oppose the change to the fcc phase solely depend on the shear constant $C^{\prime}$, i.e. a small $C^{\prime}$ alone already indicates low potential barriers for a bcc $\rightarrow$ fcc transition [23].

\subsection{Consequences of the martensitic transition}

The dynamics of the high temperature bec phases of the group 3 and 4 metals is dominated by a valley of phonons of unusual low energy and strong damping along $[\xi \xi 2 \xi]$ and $[\xi \xi 0]$ propagation. The example demonstrating this for $\gamma$-La is depicted in Fig. 8. Along this valley in the $4 \operatorname{dim}$. $\vec{Q}-\omega$ space, we find all the large amplitude fluctuations one needs to transform the bcc lattice to $\omega$, hcp, fcc or related structures like $7 \mathrm{R}, 9 \mathrm{R}, \mathrm{d}$-hcp etc... Which of these locks in at the transition can hardly be determined from the phonons themselves, it depends on subtle details of the free energy of the product phase.

\subsubsection{What stabilizes bcc?}

The other question as to why bcc is stable at high temperature although considerable fluctuations towards close packed structures are observed is easier to answer. Knowing the phonon dispersions in the parent and product phase, vibrational entropy changes $\Delta S_{\mathrm{v}}$ at the transition temperature can be calculated rather precicely in quasiharmonic approximation [24]. A comparison with the known excess enthalpy $\Delta S_{\text {tot }} \cdot T_{M}$ of the bcc $\rightarrow$ close packed transition - see Table 1 - shows that roughly $2 / 3$ of the excess enthalpy is due to the vibrational entropy. The latter is dominated by the low-energy phonons. The remaining $\Delta S_{\text {tot }}-\Delta S_{v}$ $=\Delta S_{\mathrm{el}}$ can then be ascribed to the difference in electronic entropy $\Delta \mathrm{S}_{\mathrm{el}}$. Consequently, the role of these transverse energy phonons is two fold: they are due to the instability of the bcc lattice towards a martensitic transition but in the same instance bcc is stabilized due to their contribution to the vibrational entropy.

Table 1: Vibrational $\Delta S_{\mathrm{v}}$ and total entropy changes $\Delta S_{\text {tot }}$ at the bcc $\rightarrow$ close packed transition

$\begin{array}{cccc} & \mathrm{T}_{0}(\mathrm{~K}) & \Delta \mathrm{S}_{\mathrm{v}}\left(\mathrm{k}_{\mathrm{B}} / \text { atom }\right) & \Delta \mathrm{S}_{\text {tot }}\left(\mathrm{k}_{\mathrm{B}} / \text { atom }\right) \\ \mathrm{Ti} & 1156 & 0.29 & 0.42 \\ \mathrm{Zr} & 1135 & 0.26 & 0.40 \\ \mathrm{La} & 1138 & 0.26 & 0.30\end{array}$

These considerations are not limited to the elementary systems. Ultrasonic and neutron measurements on a series of $\mathrm{Cu}$ based tertiary shape memory alloys [22,25] show similar low energy phonons as in the 
group 3 and 4 metals. In particular large anisotropy parameters $A$ are found. Hence it can be concluded that in the $\mathrm{Cu}$ based alloys also, the martensitic transformation is practically a pure vibrational driven transition [25].

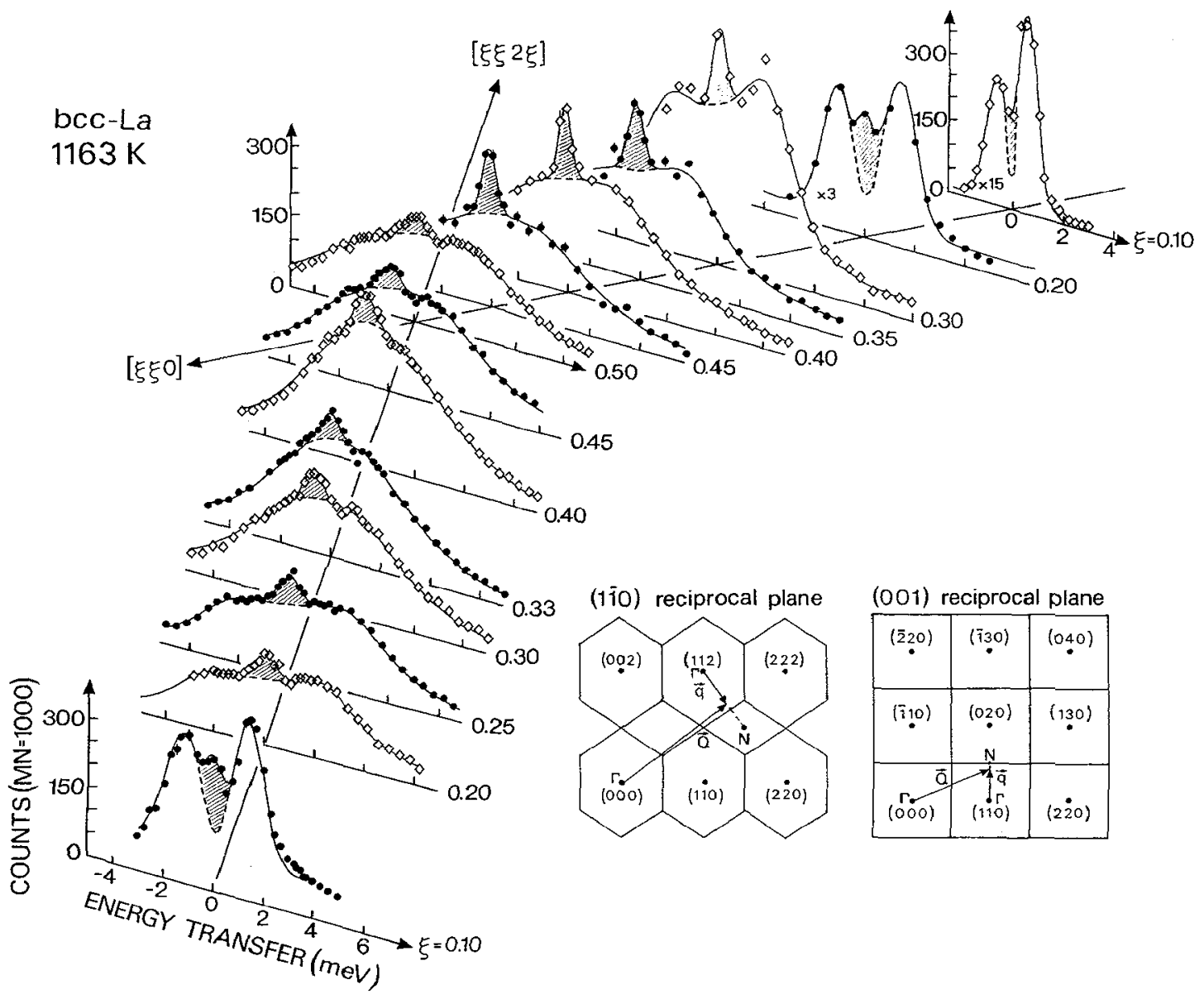

Fig. 8 Low energy and damped phonons along $\mathrm{T}_{1}[\xi \xi 2 \xi]$ and $\mathrm{T}_{1}[\xi \xi 0]$ in $\gamma$-La [14].

\subsubsection{Defect driven condensation of static displacements}

Static displacement towards the product phase but still within the parent phase have been reported in many alloyed systems. Prominent examples are the $\omega$ phase alloy $\mathrm{Zr}_{0.8} \mathrm{Nb}_{0.2}$ [26] and the shape memory alloys $\mathrm{Ni}_{1-\mathrm{x}} \mathrm{Al}_{\mathrm{x}}$ [27] and $\mathrm{Ni}_{0.5} \mathrm{Ti}_{0.5}$ [28]. It was one of the surprises of the neutron measurements in $\beta-\mathrm{Zr}$ and other pure metals that no truly elastic scattering has been observed at these points in reciprocal space where the phonons are overdamped. From this arises the question to which extent these static precursors of the martensitic transition are defect related or alternatively generic to the transition .

A first answer is quickly given: A considerable part of what has been reported in literature about elastic precursors related to martensitic transitions is simply due to heterogenous co-existence of product and parent phase. Reasons for this co-existence might be temperature gradients, concentration gradients, impurity driven phase separation, thermal non-equilibrium conditions etc ... A good example of how gaseous impurities are the driving force for a strongly temperature dependent segregation has been reported for $\alpha$-precipitates in $\beta-\mathrm{Zr}$ due to Oxygen and Nitrogen contamination [29]. 
As stated before diffuse scattering at zero energy transfer in pure samples of the bcc high temperature phase is of inelastic nature. Upon alloying with impurities the elastic intensity increases with respect to the neighbouring inelastic intensity. The difference between both is then due to truly elastic diffuse scattering $[11,30]$. In reciprocal space this additional elastic diffuse intensity is found exactly along the valley of the low energy and strongly damped phonons. Fig. 9 gives the example of $\beta$-Zr alloyed with 1.5 at \% Co [30]. Always similar diffuse patterns are observed for impurities of a so different nature as $\mathrm{O}, \mathrm{N}$, $\mathrm{Co}$, or $\mathrm{Nb}$ [31]. Only the strength of the effect changes.
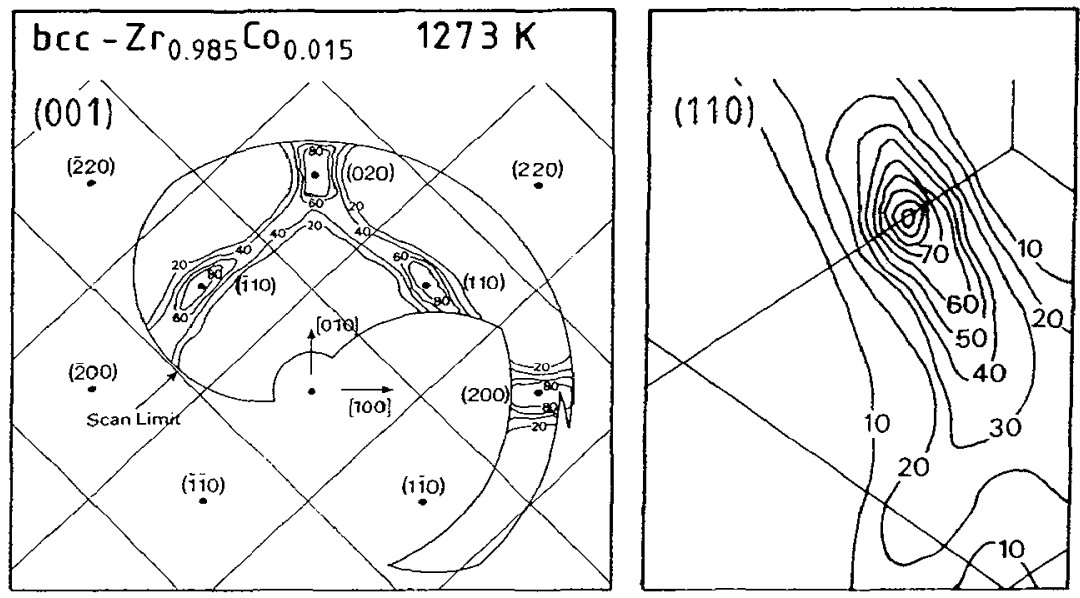

Fig. 9 "Truly" diffuse scattering in bcc-Zr due to alloying of 1.5 at \% Co [30].

In the pure elements the displacements towards the close packed structures are of purely dynamical nature. The existence of diffuse elastic scattering in the presence of point defects indicates that parts of the fluctuations freeze to static displacements. Careful analysis of the diffuse intensity distribution for instance by Kawasaki force models show that the displacement field around the solutes is a superposition of all the displacements for which low energy phonons are observed [30]. Assignments like diffuse scattering due to $\omega$-embryos are oversimplifications, they arise from measurements of the diffuse scattering only in the [110] plane and oversee its 3-dimensional character.

The absense of elastic precursors is not limited to transitions in monoatomic pure samples. The measurements on a series of $\mathrm{Cu}$ based shape memory alloys by Manosa and co-workers [32] confirm the observation that diffuse elastic scattering which might be related to the martensitic transition can be avoided if the alloys are carefully prepared and annealed.

Keeping in mind that at least in the examples cited above diffuse elastic scattering is rather a consequence of defects in the parent phase, than a property inherent to the martensitic transition, a szenario which describes the martensitic transition as a uniform transformation driven by a free energy difference seems to be appropriate. Heterogenous nucleation at defects might play a role for the transition in cases where they are present, however, they are certainly not a necessary prerequisit for the martensitic transformation.

\subsubsection{Anomalous diffusion}

At half the melting temperature $T_{m}$ self diffusities $D(T)$ in the elementary bcc structures vary by roughly 8 orders of magnitude [33]. Elements with martensitic transitions show the highest diffusivity rate whereas the most stable bcc metals of group 6, Cr, Mo, and $\mathrm{W}$ have particularly low diffusivities. Direct [34] and indirect measurements [33] of the diffusion mechanism lead to the conclusion that despite the great variety in $\mathrm{D}$, one and the same mechanism is responsible for the self diffusion in all bcc metals, namely the diffusion via nearest neighbour vacancies. The question, why $D\left(1 / 2 \mathrm{~T}_{\mathrm{m}}\right)$ differs so much, if the same mechanism is valid, remains. Of course it is appealing to suspect a relation between the tendency of the 
bcc metals to undergo phase transitions and their unusual diffusivities. In Fig.10 the possible connection is shown: The $L 2 / 3(111)$ as well as the $T_{1} 1 / 2(110)$ phonon displace the lattice in such a way that atoms are pushed into the direction of an eventually present nearest neighbour vacancy. As argued before, these displacements are of large amplitude in those metals which exhibit "weak" bcc structures, i.e. the phonons tell us that - at least during the harmonic part of a nearest neighbour jump - migration barriers are extraordinarily low. Of course diffusion is not only promoted by the two phonons shown in Fig. 10 but by the whole set of displacements related to the tendency of the bcc metals to undergo transitions.
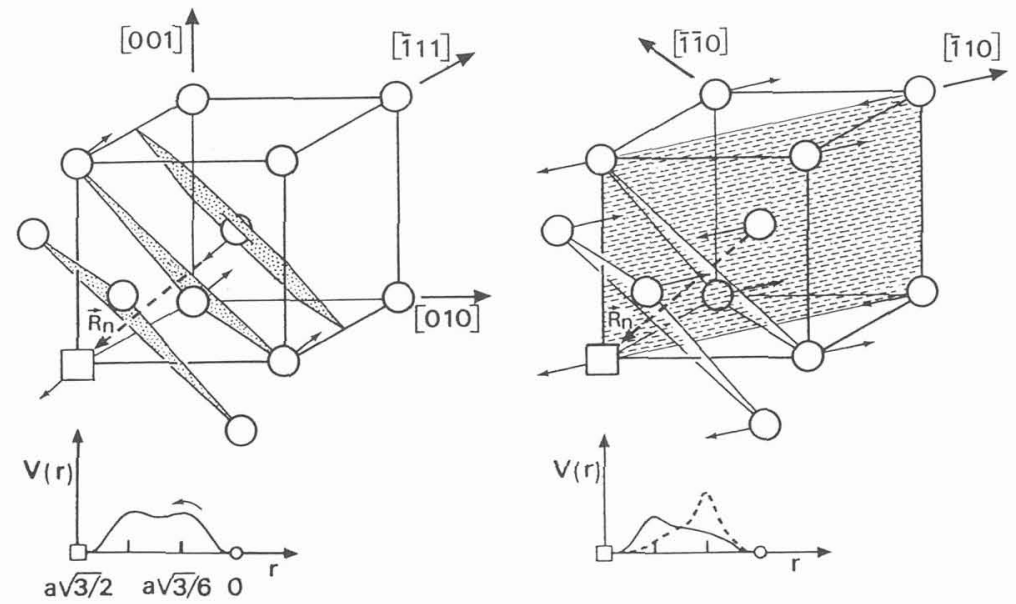

Fig.10 The displacements of the $L 2 / 3(111)$ (left) and the $T_{1} 1 / 2(110)$ phonon (right) push the centered atom in direction of an eventually present nearest neighbour vacancy. At the left a quasi-static double peak potential is seen by the migrating atom. At the right the phonon itself alters the barriers which it has to overcome during a vibrational period [33].

This idea has been worked out more quantitatively [35] and the migration barrier $\mathrm{H}^{\mathrm{m}}$ could be expressed in terms of a purely structural term $\alpha \cdot a^{2}$ characteristic for all $b c c$ phases and a term explicitely reflecting the dynamical response of the actual lattice

$$
H^{m}=\alpha \cdot a^{2}\left(\int \frac{Z(\omega)}{M \omega^{2}} d \omega\right)^{-1}
$$

Here ' $a$ ' is the lattice constant, $M$ the atomic mass and the weighting of the phonon density of states $Z(\omega)$ by $\omega^{-2}$ guarantees the dominating influence of the low energy phonon anomalies.

This model which relates the diffusion anomalies in bcc metals to varying migration barriers has been very successful in explaining the different experimental facts: i) The calculated $H^{\mathrm{m}}$ is close to the measured values, ii) the pronounced chemical group systematics observed in the diffusivity and $\mathrm{H}^{\mathrm{m}}$ is reproduced and iii) migration barriers are established as temperature dependent quantities, with gradients in opposite directions for bcc metals as different as $\beta-\mathrm{Zr}$ and $\mathrm{Cr}$. Fig. 11 compares calculated and measured $\mathrm{H}^{\mathrm{m}}$ and also indicates the lattice dynamical reason for the systematic variation of $\mathrm{H}^{\mathrm{m}}$.

\section{TRANSITIONS FROM CLOSE PACKED STRUCTURES $\rightarrow$ BCC}

The most straight forward examples are the transitions of the low temperature $\alpha$ or hcp phases of the group 4 metals $\mathrm{Ti}, \mathrm{Zr}$ or $\mathrm{Hf}$ to high temperature bcc. No dynamical precursors have been found so far, which is in itself not astonishing. As known from ground state calculations [36] hcp is the structure with the lowest internal energy and as argued before bcc is mainly stabilized by vibrational entropy of the bcc phase itself. 


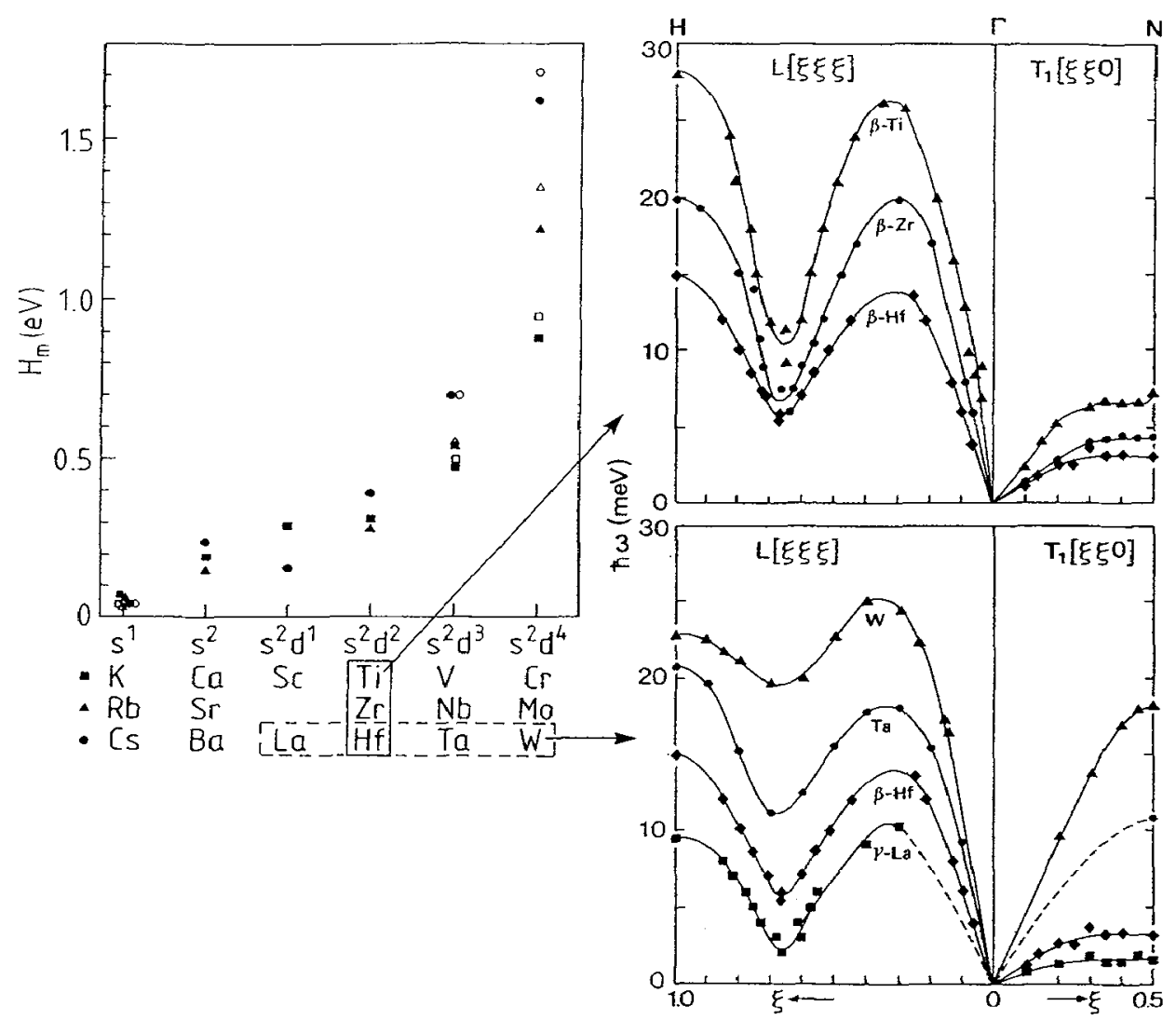

Fig. 11 Left: Migration barriers $\mathrm{H}^{\mathrm{m}}$ calculated (black sympols) according to the model of phonon controlled diffusion and compared to measured values (open symbols). Right: Explanation of the chemical group systematics by low energy phonons [35].

A more promising example could be the invar system $\mathrm{Fe}_{3} \mathrm{Pt}$. Here a strong composition and ordering dependent martensitic transition from a high temperature fcc phase to a low temperature bcc phase is found [36]. According to the Nishiyama-Wassermann rule

$$
(111)_{\mathrm{fcc}} \|(110)_{\mathrm{bcc}} \text { and }[1 \overline{1} 0]_{\mathrm{fcc}} \|[001]_{b c c}
$$

(111) $[11 \overline{2}]$ shuffles are needed to transform the fcc $\mathrm{ABCABC} \ldots$ stacking sequence to a $\mathrm{ABAB}$... stacking sequence of $(110)_{\text {bcc }}$ planes. Despite several precise neutron and ultrasonic measurements particularly along the $\mathrm{T}_{[11 \overline{2}]}[\xi \xi \xi]$ branch no anomalies evidently connected to the transition could be identified [38,39]. Instead a pronounced decrease of the $T_{[1 \overline{1} 0]} 1 / 2(110)$ phonon similar to that observed in the bcc metals was found in the austenitic fcc phase [38]. Whether the displacements of this phonon are related to the transition remains to be solved.

\section{THE FCC $\rightarrow$ HCP TRANSITION}

$\mathrm{Co}$ is an often discussed example. At $\mathrm{T}_{0}=695 \mathrm{~K}$ the high temperature phase of fcc-Co transforms to the low temperature hep structure. For the transition closest packed stacking sequences have to change from $A B C A B C \ldots$ to $A B A B \ldots$ Several models have been proposed for the transition, for instance Shapiro and Moss [40] postulate the coherent superposition of two (111) [11 $]_{\mathrm{fce}}$ shuffles with wavelength of $\lambda_{1}=3 \mathrm{~d}_{111}$ and $\lambda_{2}=6 \mathrm{~d}_{111}$, and more recently Folkins and Walker [41] showed a transition path which is the sum of a modulation and a strain. However, experiments so far are at variance to all these explanations. Firstly, most of the experiments were done on alloyed crystals [40] and secondly, recent careful measurements 
along $\mathbf{T}_{[11 \overline{2}]}[\xi \xi \xi]_{\mathrm{fcc}}$ in pure Co in the fcc as well as in the hep phase $[42,43]$ did not reveal any dynamical anomaly related to the transition. The only experimental hint that Co indeed transforms by shearing (111) fce planes comes from relatively low energies of the $\mathrm{T}[\xi \xi \xi]$ phonon branch at short as well as long wavelengths.

A very pronounced phonon anomaly, has been found in fcc or $\beta$-La which transforms upon cooling at $609 \mathrm{~K}$ to d-hcp. At $\xi=0.42$ the dispersion along $\mathrm{T}[\xi \xi \xi]$ branch exhibits a strong temperature dependent dip [44]. However, this example might not be conclusive. The anomaly is only present in metastable $\beta$-La below room temperature.

\section{SUMMARY}

From phonon measurements, shuffles have been identified which promote the transition from bcc towards $\omega$, hcp or fcc. With the exception of the bcc $\rightarrow \omega$ transition the shuffles alone cannot transform the lattice, further homogenous strains are needed. In none of the cited examples elastic precursors or defects play any obvious role during the transition, which favours the picture of a free energy driven uniform transformation of the entire crystal. The phonon dispersion in the transition elements of group 3 and 4 indicates low restoring forces for fluctuations not only towards one discrete close packed structure but to all of them. The one which locks in at the transition cannot be judged from the phonons alone but is rather a question of subtle details of the internal energy.

The tendency of the bcc metals to transform has severe consequences on the physical properties of these metals. Vibrations in these crystals behave liquid-like along certain directions in reciprocal space. These dynamical fluctuations promote both the stability of bcc by entropy and the transformation because they are indicative for low restoring forces. Further, self diffusion in bcc can be explained by low migration barriers, the height of which is given by the low energy phonons probing the harmonic part of the migration potential.

The situation is much less clear for the reverse transition, namely close packed $\rightarrow$ bcc. No shuffle can be identified, only low shear constants are observed. The situation is similar for the transition within closest packing. Further experiments on better defined, if possible, elementary systems and at transitions at high temperature are necessary. Whereas high temperature certainly hinders the experimental access, it is the best way to guarantee the oberservation of the martensitic transitions in thermal equilibrium and due to the higher thermal energy, pinning of the transition on defects is less probable.

\section{REFERENCES}

[1] Young D.A., Phase diagrams of the elements (University of California Press, Berkeley, 1991)

[2] Delaey L., in Phase Transformations, Material Sience and Technology Vol.5, Vol ed. P. Haasen (VCM Weinheim 1991) pp.339

[3] Petry W., Flottmann T., Heiming A., Trampenau J., Alba M. and Vogl G., Phys. Rev. Lett. 61 (1988) 722

[4] Schwarz W., Blaschko O. and Gorgas I., Phys. Rev. B 44 (1991) 6785; Smith H.G., Berliner R., Jorgensen J.D., Nielsen M. and Trivisonno J., Phys. Rev. B 41 (1990) 1231

[5] Schwarz W., Blaschko O. and Gorgas I., Phys Rev. B 46 (1992) 14448; Smith H.G., Berliner R., Jorgensen J.D and Trivisonno J., Phys. Rev. B 43 (1991) 4524; Blaschko O. and Krexner G., Phys. Rev. B 30 (1984) 1667

[6] Ye Y.Y., Chan C.T., Ho K.-M. and Harmon B.N., Int.J. Supercomput. Appl.4, (1991) 111

[7] Gooding R.J. and Krumhansl J.A., Phys. Rev. B 38 (1988) 1695; Gooding R.J., Ye Y.Y., Chan C.T., ho K.-M. and Harmon B.N., Phys. Rev. B $\mathbf{4 3}$ (1991) 13626

[8] Stassis C., Zarestky J., Wakabayachi N., Phys. Rev. Lett. 41 (1978) 1726; Stassis C.and Zarestky J., Solid State Comm. 52 (1984) 9

[9] Flottmann Th., Petry W., Serve R. and Vogl G., Nucl. Instr. \& Methods A 260, (1987) 165

[10] Petry W., Heiming A., Trampenau J., Alba M., Schober H.R. and Vogl G., Phys. Rev. B 43 (1991) 
10933

[11] Heiming A., Petry W., Trampenau J., Alba M., Herzig C., Schober H.R. and Vogl G., Phys. Rev. B 43 (1991) 10948

[12] Trampenau J., Heiming A., Petry W., Alba M., Herzig C., Miekeley W.and Schober H.R, Phys. Rev. B 43 (1991) 10963

[13] Petry W., Trampenau J., Herzig C., Phys. Rev. B 48 (1993) 881

[14] Güthoff F., Petry W., Stassis C., Heiming A., Hennion B., Herzig C.and Trampenau J., Phys. Rev. B 47 (1993) 2563

[15] Dubos O., Petry W., Trampenau J. and Hennion B., unpublished

[16] Glyde H.R., Can. J. Phys. 52 (1974) 2281

[17] Ho K.-M., Fu C.-L. and Harmon B.N., Phys. Rev B 28 (1983) 6687

[18] Burgers W.G., Physica 1 (1934) 561

[19] Lingard P.-A. and Mouritsen O.G., Phys. Rev. Lett. 57 (1986) 2458

[20] Krumhansl J.A. and Gooding R.J., Phys. Rev. B 39 (1989) 3047

[21] Chen Y., Ho K.-M. and Harmon B.N., Phys. Rev. B 37 (1988) 283

[22] Guenin G., Rios Jara D., Morin M., Delaey L., Pynn R. and Gobin P.F., J. Physique (Paris), Colloque 43 (1982) C4-597

[23] Gooding R.J., private communication

[24] Schober H.R. and Petry W. in Structure of Solids, Material Science and Technology, Vol 1, Vol. ed. V. Gerold (VCH-Weinheim 1993) pp.289

[25] Planes A., Manosa L., Rios-Jara D. and Ortin J., Phys. Rev. B 45 (1992) 7633; Manosa L., Planes A., Ortin J. and Martinez B., Phys. Rev. B 48 (1993) 3611

[26] Noda Y., Yamada Y. and Shapiro S.M., Phys. Rev. B 40 (1989) 5995

[27] Shapiro S.M., Yang B.X., Noda Y., Tanner L.E. and Shryvers D., Phys. Rev B 44 (1991) 9301

[28] Müllner M., Tietze H., Eckold G. and Assmus W. in Proc. Int. Conf. Martensitic Transformations, ICOMAT 86, Nara (Japan), ed. I. Tamura (The Japan Inst. of Metals 1987) pp.159

[29] Heiming A., Petry W., Trampenau J., Alba M., Herzig C. and Vogl G., Phys. Rev. B 40 (1989) 11425

[30] Heiming A., Petry W., Vogl G., Trampenau J., Schober H.R., Chevrier J. and Schärpf O., Z. Phys. $B$ 85 (1991) 239

[31] Petry W., Phase Transitions 31 (1991) 119

[32] Manosa L., Zarestky J., Lograsso T., Delaney D.W. and Stassis C., Phys. Rev. B 48 (1993) 15708

[33] Petry W., Heiming A., Herzig C. and Trampenau J., Defect \& Diffusion Forum 75 (1991) 211

[34] Vogl G., Petry W., Flottmann T. and Heiming A., Phys. Rev. B 39 (1989) 5025

[35] Schober H.R., Petry W. and Trampenau J., J. Phys. Condens. Matter 4 (1992) 9321

[36] Pettifor D.G.. in Structure of Solids Material Science and Technology, Vol 1, Vol. ed. V. Gerold (VCH-Weinheim 1993) pp.61

[37] Wassermann E.F. in Ferromagnetic Material Vol 5, eds K.H. Buschow, E.P. Wollfarth (North Holland, Amsterdam 1990) pp.240

[38] Noda Y. and Endoh Y., J. Phys. Soc. Japan 57 (1988) 4225

[39] Kawald U., Schulenberg P., Bach H., Pelzl J., Eckold G. and Saunders A., J. Appl. Phys. 70 (1991) 6537

[40] Shapiro S.M. and Moss S.C., Phys. Rev. B 15 (1977) 2726

[41] Folkins I. and Walker M.B.., Phys. Rev. Lett. 65 (1990) 127

[42] Frey F., Prandl W., Schneider J., Zeyen C. and Ziebeck K., J. Phys. F 9 (1979) 603

[43] Petry W., Frey F., Strauß R. and Shapiro S.M., unpublished

[44] Stassis C., Smith G.S., Harmon B.N., Ho K.-M. and Chen Y., Phys. Rev. B 31 (1985) 6298 